\title{
TERAPIA COMUNITÁRIA INTEGRATIVA: ANÁLISE ACERCA DO PERFIL DAS PRODUÇÕES CIENTÍFICAS EM CONGRESSOS BRASILEIROS
}

\author{
Simone de Moura AGUIAR ${ }^{1}$ \\ Elisângela Braga de AZEVEDO² \\ Lorena de Farias Pimentel COSTA ${ }^{3}$ \\ Jeferson Barbosa SILVA \\ Camila Carla Dantas SOARES ${ }^{5}$ \\ Maria de Oliveira Ferreira FILHA ${ }^{6}$
}

\begin{abstract}
${ }^{1}$ Enfermeira Graduada pela Faculdade de Ciências Médicas de Campina Grande. E-mail: Simonemaguiar@hotmail.com

${ }^{2}$ Enfermeira. Doutoranda pelo Programa de Pós-Graduação em Enfermagem da Universidade Federal da Paraíba PPGEnf/UFPB. Professora do Curso de Enfermagem da Faculdade de Ciências Médicas. Membro do Grupo de Estudos e Pesquisas em Saúde Mental Comunitária - GEPSMEC/UFPB. E-mail: elisaaz@ terra.com.br

${ }^{3}$ Enfermeira. Mestranda pelo Programa de Pós-Graduação em Enfermagem da Universidade Federal da Paraíba PPGEnf/UFPB. Membro do Grupo de Estudos e Pesquisas em Saúde Mental Comunitária - GEPSMEC/UFPB. E-mail: lorenafarias@outlook.com

${ }^{4}$ Enfermeiro. Graduado pela Universidade Federal de Campina Grande - UFCG. E-mail: jeferson_barbosa@hotmail.com

${ }^{5}$ Enfermeira. Graduada pela Universidade Federal de Campina Grande - UFCG. E-mail: camilacarla.soares@hotmail.com
\end{abstract}

${ }^{6}$ Enfermeira. Doutora em Enfermagem pela Universidade Federal do Ceará - UFC. Professor Adjunto IV da Universidade Federal da Paraíba - UFPB. Líder do Grupo de Estudos e Pesquisas em Saúde Mental Comunitária GEPSMEC/UFPB. E-mail: marfilha@yahoo.com.br

Recebido em: 03/09/2013 - Aprovado em: 20/12/2013 - Disponibilizado em: 15/01/2014

\section{RESUMO}

Introdução: A Terapia Comunitária Integrativa configura-se como uma tecnologia leve de cuidado eficaz na prevenção do adoecimento psíquico nas estratégias de cuidado do Sistema Único de Saúde e vem ganhando destaque nas produções científicas devido a sua eficácia e reconhecimento Objetivos: analisar de forma comparativa o perfil de produção científica entre os I e VI Congressos Brasileiros de Terapia Comunitária Integrativa (CBTCI). Especificamente, avaliar o enfoque dado nos estudos; as experiências estão sendo realizadas no Brasil e o perfil dos trabalhos enviados ao CBTCI; Conhecer em quais grupos populacionais estão sendo desenvolvidas as rodas de TCI, os tipos de estudos e a Região mais pesquisada. Metodologia: Trata-se de uma pesquisa compreensiva, interpretativa, comparativa, de abordagem quantitativa, realizada de fevereiro a novembro de 2012. Com amostra composta por todos os trabalhos publicados nos Anais dos I e VI CBTCI, tendo seus principais resultados discutidos comparativamente, utilizando a literatura vigente. Resultados e Discussão: Percebeu-se que nos anais investigados os profissionais e docentes brasileiros tiveram maior porcentagem de submissões, porém, no VI visualiza-se trabalhos da argentina, Uruguai e Chile. Quanto ao enfoque dado nos estudos, saúde e educação foram às áreas mais pesquisadas e os profissionais optaram por encaminhar relatos de experiências com grupos mistos, seguidos de jovens e adultos. Considerações Finais: Embora a TCI seja considerada uma prática recente, os profissionais que a desenvolvem, tem buscado através dos CBTCI, divulgar e publicar suas experiências práticas com a TCI para os demais profissionais.

Palavras-chave: Produção científica. Terapia Comunitária. Pesquisa. Anais. Congressos.

\section{ABSTRACT}

Introduction: Community Therapy Integrative configured as a light technology for effective care in the prevention of mental illness in the strategies of care Health System and is gaining prominence in scientific productions due to its effectiveness and recognition Objectives: to analyze comparatively the profile scientific production between I and VI Brazilian Congress of Integrative Community Therapy (CBTCI). Specifically, evaluate the approach taken in the 
studies; experiments are being conducted in Brazil and the profile of the work sent to CBTCI; Knowing in which population groups are being developed wheels TCI, the types of studies and most researched Region. Methodology: This is a comprehensive research, interpretive, comparative, quantitative approach, performed from February to November 2012. With a sample of all papers published in the Proceedings of the I and VI CBTCI, having its main results discussed comparatively, using the current literature. Results and Discussion: It was noted that the annals investigated the pros and Brazilian teachers had the highest percentage of submissions, however, the VI displays are works of Argentina, Uruguay and Chile. As for the approach taken in the studies, health and education were the most researched areas and professionals chose to forward reports of experiments with mixed groups, followed by youth and adults. Conclusions: Although the TCI is considered a recent practice, the professionals who develop, has sought through CBTCI, distribute and publish their practical experiences with TCI to other professionals.

Keywords: Scientific production. Community Therapy. Search. Annals. Congress.

\section{INTRODUÇÃO}

Regido pelas Portarias Ministeriais n. 971, em 03 de maio e n. 1.600, de 17 de julho, ambas do ano de 2006, foi aprovado pelo Conselho Nacional de Saúde, a Política Nacional de Práticas Integrativas e Complementares do SUS (PNPIC-SUS) em que é possível encontrar novas modalidades do cuidar baseados em um modelo preventivo e comunitário, destacando-se a Terapia Comunitária Integrativa (TCI) como uma pratica de cuidar não alopática que visa construir redes de apoio na comunidade, com intuito de complementar a atenção primária em saúde mental

A TCI configura-se em uma tecnologia leve de cuidado, considerada mais um instrumento inovador de trabalho na saúde mental, levando à comunidade a oportunidade de expressar sentimentos e descrever situações promotoras do adoecimento mental, possibilitando melhora na saúde, bem como a redução das perturbações psicossomáticas (CORDEIRO et. al., 2011).

Com as constantes transformações nas políticas públicas em saúde mental no Brasil, é cada vez mais perceptível a adesão dos profissionais as novas formas de cuidar. A TCI se destaca por oferecer um espaço aberto para usuários, familiares e profissionais, cujo objetivo, é construir vínculos solidários e restaurar a autoestima, por meio da construção de redes de apoio social e empoderamento, tanto individual como coletivo (BARRETO, 2008).

A proposta se fundamenta em abordar e acolher o sofrimento humano gerado pelos problemas sociais da modernidade, estimulando o fortalecimento de redes comunitárias, prevenindo a evolução de problemas familiares e sociais através da valorização da herança cultural, do resgate das origens e das raízes históricas do povo (CORDEIRO et al., 2011).

Com o avanço das ciências e maior estimulo ao desenvolvimento técnicocientifico, os eventos acadêmicos vêm se destacando como fonte eficaz para a troca de informações advindas das praticas profissionais cotidianas ou resultados de pesquisas, uma vez que promovem discussões que trazem ao conhecimento público 
resultados de novos estudos e tecnologias (LACERDA; WEBER; PORTO, 2008).

De acordo com Mancia e Padilha (2006) os congressos são espaços apropriados para se realizarem debates visando à colaboração ativa dos participantes para a discussão de novos paradigmas para as profissões, para que, desta forma possa-se contribuir para o desenvolvimento das ciências, estabelecendo, atualizando e consolidando em inúmeras práticas e tecnologias.

Com o crescimento da utilização da TCI como método de prevenção e promoção de saúde, a prática desperta cada vez mais o interesse de pesquisadores, por se mostrar eficaz no fortalecimento da rede de cuidado na atenção básica, que leva à melhoria da assistência e qualidade de vida individual e comunitária.

Partindo desse pressuposto, surgiu como questionamento que norteou o estudo: quais as tendências de pesquisas que foram publicadas nos anais do I e no VI Congressos Brasileiros de Terapia Comunitária Integrativa (CBTCI)?

E para responder tal questionamento emergiu como objetivo, analisar de forma comparativa o perfil de produção científica dos I e VI CBTCI, realizado nos anos de 2003 e 2011. Buscando-se conhecer o enfoque abordado nos congressos; Identificar quais pesquisas estão sendo realizadas no Brasil e qual o perfil destas; Apontar em quais grupos populacionais os terapeutas/profissionais desenvolvem as rodas de Terapia Comunitária Integrativa (TCI), bem como qual região apresenta maior índice de experiências.

\section{PERCURSO METODOLÓGICO}

Trata-se de uma pesquisa compreensiva, interpretativa e comparativa com abordagem quantitativa. A população foi constituída por trabalhos publicados nos Anais dos CBTCI, tendo como amostra os trabalhos apresentados e publicados nos Anais dos I e VI CBTCI (primeiro e último congressos ocorridos) para assim, proporcionar uma análise comparativa mais consistente.

Foram levados em consideração os seguintes critérios de inclusão: trabalhos publicados nos anais do I e VI CBTCI na forma de resumo e ou trabalho na integra e que atingissem os objetivos propostos. Sendo excluídos, os que não atendessem os critérios acima citados.

Como instrumento de coleta do material, foi desenvolvido pelos autores um formulário de catalogação e aquisição dos anais impressos no acervo do Grupo de estudos e pesquisas em Saúde Mental e Comunitária (GEPSMEC- UFPB). O material foi lido individualmente no período de fevereiro a novembro de 2012, o que permitiu a abstração os dados pertinentes para atender os objetivos propostos. 
Posteriormente os dados foram agrupados em categorias e dispostos em duas tabelas, sendo uma para o I e outra para o VI CBTCI de forma a facilitar a discussão comparativa com a literatura vigente.

A pesquisa realizada foi regida pelas diretrizes emanadas na Lei 9.610 de 19 de fevereiro de 1998 que discorre sobre os direitos autorais, resguardando os nomes dos autores e ano de publicação da obra. Respeitando os anonimatos das pessoas que realizaram as pesquisas, bem como as normas para manipulação de material.

\section{RESULTADOS}

Tabela 1 - Resultados das pesquisas identificadas nos anais do congresso brasileiro de terapia comunitária de 2003.

\begin{tabular}{llll}
\hline Países que publicaram: & Frequência & Percentual(\%) \\
\hline Brasil & 24 & 88.9 \\
Não informado & 03 & 11.1 \\
\hline Total & 27 & 100 \\
\hline Região do Brasil & & \\
\hline Sudeste & 06 & 22.22 \\
Sul & 07 & 25.93 \\
Nordeste & 06 & 22.22 \\
Centro oeste & 05 & 18.52 \\
Não informado & 03 & 11.11 \\
\hline Total & 27 & 100 \\
\hline Área de enfoque dos & & \\
estudos: & & 7.41 \\
\hline Direitos humanos & 01 & 3.70 \\
Educação & 04 & 62.96 \\
Saúde & 17 & \\
Saúde e direitos & 02 & \\
humanos & 02 & \\
Saúde e educação & & \\
\hline
\end{tabular}

\begin{tabular}{|c|c|c|}
\hline Saúde e Sociedade & 01 & 3.70 \\
\hline Total & 27 & 100 \\
\hline \multicolumn{3}{|l|}{ Tipo de Estudo } \\
\hline Relato de Experiência & 19 & 70.37 \\
\hline Pesquisa & 05 & 18.51 \\
\hline Artigo de reflexão & 03 & 11.11 \\
\hline Total & 27 & 100 \\
\hline \multicolumn{3}{|l|}{$\begin{array}{l}\text { Participantes da } \\
\text { Pesquisa }\end{array}$} \\
\hline Usuários & 02 & 3.28 \\
\hline \multicolumn{3}{|l|}{ ESFlcomunidadelCAPS } \\
\hline $\begin{array}{l}\text { Terapeutas } \\
\text { comunitários }\end{array}$ & 01 & 1.64 \\
\hline Jovens\adultos & 06 & 9.83 \\
\hline Grupos & 09 & 14.75 \\
\hline \multicolumn{3}{|l|}{ (Crianças/ adolescentes } \\
\hline \multirow{2}{*}{\multicolumn{3}{|c|}{$\begin{array}{l}\text { /jovens/ } \\
\text { idosos): }\end{array}$}} \\
\hline & & \\
\hline Mulheres & 01 & 1.64 \\
\hline Adultos & 03 & 4.92 \\
\hline Não informado & 05 & 8.20 \\
\hline Total & 61 & 100 \\
\hline \multicolumn{3}{|l|}{ Pesquisadores } \\
\hline Docentes & 05 & 18.51 \\
\hline Terapeuta comunitário & 09 & 33.33 \\
\hline Profissionais da assist./ & 09 & 33.33 \\
\hline \multicolumn{3}{|l|}{ Não terapeuta } \\
\hline Não informado & 04 & 14.81 \\
\hline Total & 27 & 100 \\
\hline
\end{tabular}

Fonte: Anais do I congresso de Terapia Comunitária integrativa - 2003.

A tabela acima revela que dentre os trabalhos encaminhados para o congresso de TCI de 2003, 24 (88,9\%) foram de origem brasileira. Com base no enfoque regional brasileiro, os encaminhamentos mais significativos de trabalhos foram das regiões 
Sul 07 (25,93\%), Sudeste $06 \quad(22,22 \%)$, Nordeste $06(22,22 \%)$.

No que diz respeito ao enfoque temático do trabalho, percebeu-se que a área da saúde apareceu em maior quantidade, sendo encontradas em 17 (62,96\%) trabalhos, seguida por educação com 04 (14,81\%). Identificou-se que o tipo de metodologia mais usada nas pesquisas estudadas foram relatos de experiência, compondo 19 (70,37\%) estudos, seguido por pesquisa de campo 05 $(18,51 \%)$ e artigo de reflexão $03(11,11 \%)$.

Quando avaliado o perfil dos pesquisadores, notou-se que $09(33,33 \%)$ possuíam formação em TCI, os profissionais da Assistência/ Não Terapeutas eram 09 $(33,33 \%)$, os docentes $05(18,51 \%)$ e não informados $04(14,81 \%)$.

Tabela 2 - Resultados das pesquisas identificadas nos anais do congresso brasileiro de terapia comunitária de 2011.

\begin{tabular}{lll}
\hline Países que publicaram & Frequência & Percentual(\%) \\
\hline Brasil & 42 & 68.85 \\
Argentina & 01 & 1.64 \\
Uruguai & 01 & 1.64 \\
Chile & 01 & 1.64 \\
Não informado & 16 & 26.22 \\
\hline Total & 61 & 100 \\
\hline Região do Brasil & & \\
\hline Sudeste & 22 & 36.1 \\
Norte & 04 & 0.6 \\
Nordeste & 13 & 21.31 \\
Centro oeste & 02 & 3.28 \\
Outro países & 01 & 1.64
\end{tabular}

\begin{tabular}{|c|c|c|}
\hline Não informado & 19 & 31.14 \\
\hline Total & 61 & 100 \\
\hline \multicolumn{3}{|l|}{$\begin{array}{l}\text { Área de enfoque dos } \\
\text { estudos }\end{array}$} \\
\hline Direitos humanos & 03 & 4.92 \\
\hline Educação & 06 & 9.83 \\
\hline Saúde & 39 & 63.93 \\
\hline Direitos humanos & 01 & 1.64 \\
\hline Saúde e educação & 07 & 11.48 \\
\hline Religião & 01 & 1.64 \\
\hline Cultura & 01 & 1.64 \\
\hline Não informado & 03 & 4.92 \\
\hline Total & 61 & 100 \\
\hline \multicolumn{3}{|l|}{ Tipo de Estudo } \\
\hline Relato de Experiência & 49 & 80.32 \\
\hline Pesquisa & 07 & 11.47 \\
\hline Artigo de reflexão & 02 & 3.28 \\
\hline Revisão Bibliográfica & 03 & 4.92 \\
\hline Total & 61 & 100 \\
\hline \multicolumn{3}{|l|}{ Participantes da Pesquisa } \\
\hline Usuários & 08 & 13.11 \\
\hline \multicolumn{3}{|l|}{ ESFlcomunidadelCAPS } \\
\hline Terapeutas comunitários & 05 & 8.2 \\
\hline Grupo tabagista & 01 & 1.64 \\
\hline $\begin{array}{l}\text { Grupos mistos (Crianças/ } \\
\text { adolescentes/adultos/idosos) }\end{array}$ & 19 & 31.14 \\
\hline Adolescentes/ Estudantes: & 05 & 8.2 \\
\hline \multicolumn{3}{|l|}{ Gestantes } \\
\hline Presidiários & 01 & 1.64 \\
\hline Mulheres & 01 & 1.64 \\
\hline Idosos & 02 & 3.28 \\
\hline Hipertensos & 01 & 1.64 \\
\hline Profissionais & 08 & 13.11 \\
\hline Não informado & 09 & 14.75 \\
\hline Total & 61 & 100 \\
\hline \multicolumn{3}{|l|}{ Pesquisadores } \\
\hline Docentes & 19 & 31.14 \\
\hline Discentes de Graduação & 04 & 6.55 \\
\hline Discente Mestrado & 01 & 1.64 \\
\hline Terapeuta comunitário & 06 & 9.83 \\
\hline Profissionais da assist. Não & 26 & 42.62 \\
\hline
\end{tabular}


terapeuta

\begin{tabular}{lll} 
Não informado & 05 & 8.20 \\
\hline Total & 61 & 100
\end{tabular}

Fonte: Anais do VI Congresso de Terapia Comunitária integrativa - 2011.

Notou-se que um maior número de países submeteram trabalhos para o CBTCI de 2011, dentre eles $42(68,85 \%)$ foram de linhagem brasileira, seguidos por $01(1,64 \%)$ da Argentina, 01 (1,64\%) de Uruguai, 01 $(1,64 \%)$ de Chile.

Os dados encontrados nos anais do congresso quando divididos por regiões brasileiras, mostram que a região Sudeste obteve maior número de publicações com 22 $(36,1 \%)$, seguida pela região Nordeste com 13 $(21,31 \%)$.

Com relação às áreas de enfoque dos estudos, a da saúde apareceu em 39 (63,93) trabalhos, saúde e educação em 07 (11,48\%). Percebeu-se que dentre os autores os profissionais da Assistência/ não Terapeuta representaram $26(42,62 \%)$ autores, 19 $(31,14 \%)$ eram docentes, $06(9,83 \%)$ eram terapeutas comunitários, 04 (6,55\%) discentes de graduação, $01(1,64 \%)$ era discente de mestrado e $05(8,20 \%)$ não foram informados.

Quanto aos tipos de metodologias utilizadas, o relato de experiência foi utilizado em $49(80,32 \%)$ trabalhos, seguido pelas pesquisas de campo com 07 (11,49\%), artigos de revisão bibliográfica com 03 (4,92\%) e artigos de reflexão com 02 (3,28\%).

\section{DISCUSSÃO}

Observou-se ao comparar as tabelas 1 e 2, o aumento significativo no número de trabalhos publicados nos anais, com destaque para o Brasil que possui a maior porcentagem em ambos os CBTCI avaliados, podendo essa porcentagem estar relacionada ao fato de o Brasil ser o país onde nasceu a TCI, e onde ocorreram as edições do congresso.

A crescente demanda de trabalhos entre um congresso e outro (2003/2011), nos mostra que as pesquisas na área e os resultados positivos advindos da realização das rodas de TCI tem aumentado de forma significativa o interesse do mundo acadêmico pela prática.

Outro ponto que vem sendo observado acerca dessa discussão ocorre devido às conquistas obtidas a partir da aplicabilidade da TCI no processo de inclusão social frente às populações alvo, acarretando seu crescimento, enquanto ciência e tecnologia inovadora (GUIMARÃES; FERREIRA FILHA, 2006).

Torna-se relevante enfatizar que a produção de conhecimento vem se apresentando como alavanca para o desenvolvimento da humanidade, tendo em vista a velocidade e abrangência aos quais as informações são disseminadas e utilizadas (VEIGA, 2008). Esta disseminação favorece a divulgação de novas estratégias de cuidado, bem como a difusão de práticas inovadoras e 
eficazes de promoção da saúde e prevenção do adoecimento físico e mental.

Ao comparar os dados obtidos, notouse mudanças no ranking da região brasileira que mais teve trabalhos publicados, tendo a região Sul publicado mais no I CBTCI e a Sudeste no VI CBTCI, destacando também a região Nordeste, que apresentou um elevado percentual de publicações em ambas as edições, em decorrência dos avanços e estímulos às pesquisas nesta área, grande parte desenvolvidas pelo Grupo de Estudos em Saúde Mental Comunitária da Universidade Federal da Paraíba.

Pode-se associar, contudo, o crescimento no desenvolvimento da TCI e das pesquisas relacionadas a esta temática na região Nordeste, pela mesma ter tido sua origem no Ceará, no ano de 1986 na comunidade Pirambú, uma das maiores favelas da capital Fortaleza. (JATAI; SILVA, 2012).

Por outro lado, a diversidade de regiões do país que publicam sobre a TCI pode ser explicada devido à difusão de conhecimento acerca da prática por todo o Brasil, estando atualmente implantada em dezessete estados brasileiros (JATAI; SILVA, 2012). Evidencia-se também o desenvolvimento da TCI em países como França, Suíça, Uruguai e Argentina, mostrando que a mesma tem expandido para novos espaços, consolidando-se a cada dia (BARRETO, 2008).
Através do estudo viu-se que a área da saúde tem as maiores porcentagens quando relacionada à área de enfoque dos estudos publicados nos anais dos CBTCI. Destacandose a contribuição que diversas outras áreas tem exercido, de forma a apoiar o crescimento teórico-prático, visando o aprimoramento dos profissionais e a efetivação de pesquisas.

Justifica-se também, por ser um dos principais focos de atuação da TCI, em particular na área da saúde mental e inclusão social de grupos marginalizados, em que através dela a comunidade pode expressar seus sentimentos e angústias causadoras de estresse, e adoecimento psíquico.

As rodas de TCI podem ser realizadas em diversos locais, sendo os espaços mais indicados àqueles que permitam uma interação social (OLIVEIRA; FERREIRA FILHA, 2011). Assim como foi relatado na ideia acima, a pesquisa nos mostrou que os locais com maior desenvolvimento das rodas de TCI, foram espaços acessíveis e promotores das trocas, possibilitadores da partilha de saberes e experiências vividas e relatadas de uma forma circular e horizontal, visando o alivio do sofrimento humano, através das redes.

Corroborando com esta perspectiva, Guimarães e Ferreira Filha (2006) discorrem sobre a possibilidade de desenvolvimento de rodas de TCI em diferentes níveis de atenção, a exemplos de serviços como Unidade Saúde da Família, ambulatórios e maternidade. 
Contando ainda com espaços de cunho comunitário como igrejas, escolas, associação de moradores e clubes.

Ante tais perspectivas para o desenvolvendo as rodas de TCI, pode-se inferir sobre grupos importantes que surgiram e foram citados no CBTCI de 2011 como, grupos de tabagistas, adolescentes gestantes, presidiárias e hipertensos. Neste caso, fica ainda mais clara a diversidade cultural e social em que a TCI pode estar inserida. Pode-se inferir essa afirmação em estudos publicados sobre a aplicabilidade da TCI e sua eficácia em grupos de gestantes, idosos e mulheres, o qual identificou que tal prática desenvolve nas pessoas uma melhoria na autoestima e qualidade de vida, prevenindo assim, o adoecimento psíquico, além de melhorar a capacidade resiliente das pessoas que participam das rodas (ANDRADE et al., 2010; HOLANDA; DIAS e FERREIRA FILHA, 2011).

Em outra vertente, a TCI como não se propõe a oferecer a resolução dos problemas dos participantes, pois promove, principalmente, a formação de redes de apoio solidária para que estas contribuam para o fortalecimento do sujeito, família e comunidade, para que assim, ocorra a mudança (BARRETO, 2008). O compartilhamento de experiências, impulsionam momentos de reflexão para os integrante das rodas, funcionando como mola propulsora para o (re)conhecimento do poder resiliente de cada um (ROCHA et al, 2009).

Guimarães e Ferreira Filha (2009) destacam o benefício proposto pela TCI a grupos em situação de vulnerabilidade, à medida que as histórias dos participantes deixam evidente sua corresponsabilidade pela vida a partir do momento que elevam o poder de autodeterminação, estimulando a confiança e autonomia para tomada de decisões, afirmando a importância da imagem que se tem de si, para que consiga lidar melhor com os conflitos e adversidades do cotidiano causadoras de sofrimento psíquico, de modo a superá-las com mais facilidade para alcançar seus objetivos.

Destacou-se também que, em ambos os eventos o número elevado de encaminhamentos de trabalhos por profissionais da assistência não terapeutas. Com isso, infere-se que mesmo não sendo terapeuta formados os profissionais empenham em divulga-la como uma prática de qualidade e necessária. Demonstra-se, então, que a pesquisa, segundo Guariente et al., (2006) é necessária para esses profissionais para subsidiar a busca de soluções para os problemas do cuidar. Sendo esta impulsionadora do processo de refletirexperienciar-transformar, configurando-se em uma estratégia fundamental para mudar a qualidade da assistência nos serviços prestados à população. 
Referente aos delineamentos
metodológicos das pesquisas na amostra
estudada identificou-se, que em grande parte dos trabalhos, foi utilizado relato de experiência, que é uma forma metodológica que permite a descrição de situações vivenciadas pelos autores da pesquisa.

Logo, esse estudo, leva a reflexão que os avanços científicos e tecnológicos têm proporcionado mudanças expressivas no processo de viver e desenvolver do ser humano, o qual agrega consequentemente repercussões das mais variáveis para o cotidiano do trabalho, educação, pesquisa e saúde. Assim, ao voltar o enfoque ao ciclo de vida humano, desde a concepção e nascimento até morte, torna-se imprescindível reconhecer os benefícios da ciência e da tecnologia como forma de aperfeiçoamento as práticas de cuidado à saúde (RAVELLI et al., 2009).

\section{CONSIDERAÇÕES FINAIS}

$\mathrm{Na}$ atualidade os profissionais defrontam-se com diversas formas de cuidar em saúde. A PNPIC-SUS surge neste cenário como uma forma de consolidação e estimulo ao uso práticas sociais e não alopáticas. A prática da TCI, como foi demonstrado no estudo, vem se destacando no cenário nacional e internacional por ser uma tecnologia de cuidado de baixo custo, que preenche a lacuna deixada por outras práticas, haja vista que, por meio do trabalho em grupo, possibilita-se a reabilitação e inclusão social e o desenvolvimento de ações de prevenção e promoção da saúde, em especial da saúde mental de diversos grupos inseridos na comunidade.

O estudo possibilitou visualizar o aumento no número de publicações que abordou a TCI em todas as regiões do país. Os locais que mais ocorrem às rodas de TCI foram USF, CAPS, comunidade e grupos mistos que culturalmente são espaços para encontro grupais dentro da sociedade ou mesmo naqueles com maior probabilidade de exclusão social. Logo, entende-se que quando realizadas nestes espaços, a TCI solidifica-se no modelo de atenção de base comunitária.

Acredita-se, portanto, que o estimulo a terapêuticas não farmacológicas para intervir na saúde, tem surgido como uma alavanca para disseminação das Práticas Integrativas e Complementares do SUS, levando os profissionais a incluir novas formas de cuidar em sua prática profissional.

contribuições da terapia comunitária. Texto Contexto Enferm, v. 9, n. 1, p. 129-36, 2010.
ANDRADE, F. B.; FERREIRA FILHA, M. O.; DIAS, M. D. et al. Promoção da saúde mental do idoso na atenção básica: As 
BARRETO, A. P. Terapia Comunitária passo a passo. Fortaleza: Gráfica LCR; 2008.

CORDEIRO, R. C.; AZEVEDO, E. B.; SILVA, M. S. S.; et al. Terapia comunitária integrativa na estratégia saúde da família: análise acerca dos depoimentos dos seus participantes. Revista da Universidade Vale do Rio Verde. Três Corações, v. 9, n. 2, p. 192-201, 2011. Disponível em: http://revistas.unincor.br/index.php/revistauni ncor/article/view/150. Acesso em: 10 nov 2012.

GUARIENTE, M. H. D. M.; ZAGO, M. M. F.; SOUBHIA, Z.; et al. Análise da Publicação Científica de enfermeiras assistenciais com apoio de um núcleo de pesquisa em enfermagem. Semina: Ciências Biológicas e Saúde. Londrina, v. 27, n. 1, p. 41-56, 2006.

GUIMARÃES, F. J.; FERREIRA FILHA, M. O. Repercussões da Terapia Comunitária no cotidiano de seus participantes. Rev. Eletrônica de Enfermagem. V.8, n.3, p. 404414, 2006. Disponível em: http://www.fen.ufg.br/revista/revista8_3/v8n3 a11.htm. Acesso em: 12 nov 2012.

HOLANDA, V. R.; DIAS, M. D.; FERREIRA FILHA, M. O. A Experiência da Terapia Comunitária como Estratégia de Cuidado Pré-Natal. Rev enferm UFPE on line. v. 5, n. 5, p.1129-136, 2011.

JATAI, J. M.; SILVA, L. M. S. Enfermagem e a implantação da Terapia Comunitária Integrativa na Estratégia Saúde da Família: relato de experiência. Rev. Bras. Enferm. v. 65, n. 4, p. 691-695, 2012.

LACERDA, A. L.; WEBER, C.; PORTO, M. P. et al. A importância dos eventos científicos na formação acadêmica: estudantes de biblioteconomia. Revista ACB: Biblioteconomia em Santa Catarina. Florianópolis, v. 13, n.1, p. 130-144, 2008. bras. enferm., Brasília, v. 59, n. spe, p. 432-437, 2006.

OLIVEIRA, D. S. T.; FERREIRA FILHA, M. O. Contribuição dos recursos culturais para a terapia comunitária integrativa na visão do terapeuta. Rev. Gaúcha Enferm. v. 32, n. 3, p. 524-530, 2011. Disponível em: http://www.scielo.br/scielo.php?script=sci_art text\&pid=S1983-

14472011000300013\&lng=en. Acesso em: 10 de nov 2012.

RAVELLI, A. P. X.; FERNANDES, G. C. M.; BARBORA, S. F. F. et al. A produção do conhecimento em enfermagem e envelhecimento: estudo bibliométrico. Texto \& Contexto Enferm. Florianópolis, v. 18, n. 3, p. 506-512, 2009.

ROCHA, I. A.; BRAGA, L. A. V.; TAVARES, L. M.; et al. A terapia comunitária como novo instrumento de cuidado para saúde mental do idoso. Rev Bras Enferm. v. 62, n. 5, p. 687-694, 2009. Disponível em: < http://www.scielo.br/pdf/reben/v62n5/06.pdf> Acesso em: 10 out 2012

VEIGA, K. C. G.; MENEZES, T. M. O. Produção de conhecimento em enfermagem: a (in) visibilidade da atenção à saúde do idoso. Rev Esc Enferm. São Paulo, v. 42, n. 4, p. 761-8, 2008.

MANCIA, J. R.; PADILHA, M. I. C. S. Trajetória de Edith Magalhães Fraenkel. Rev. 